\title{
Overview of precancer and goals of the meeting
}

\author{
Gary J. Kelloff \\ National Cancer Institute, Cancer Imaging Program, Bethesda, MD 20852, USA
}

The presentations and discussions at this workshop identify, discuss, and summarize the human populations at risk for cancer from epidemiologic, pathologic, genetic, and molecular biology data as a basis for application of imaging modalities to evaluate these risks. For example, data will be analyzed from the perspective of relative and absolute risk for developing cancer over a defined time interval. Risk modeling from case study examples such as the Gail risk model for breast cancer will be discussed. The existing clinical and preclinical data derived from many technologies including imaging modalities identifying early cancer, precancer lesions or predysplastic molecular lesions known to be associated with cancer risk will be discussed. Imaging modalities for early detection/diagnosis of precancer and early cancer in esophagus (Barrett's), bladder, breast, prostate, lung, colon, pancreas, ovary, head and neck, cervix and skin are considered.

These presentations review existing image-guided intervention (IGI) devices available and appropriate for intervention in precancer and early cancer. Experience with IGI in clinical and preclinical settings is discussed, and especially any outcomes data resulting from IGI, as is safety data from IGI. Existing clinical data on drug intervention or surgery in patients with precancer lesions is explored, as a basis for developing strategies that complement or replace these interventions with IGI, or use IGI in settings where drug therapy or surgery is not ongoing.

Workshop sessions on each target organ begin with presentations by keynote speakers, who consider the topics listed below with the objective of identifying precancer and early cancer settings where imaging would improve risk detection or treatment. The keynotes are followed by speakers presenting overviews on the use of imaging in the clinical setting or specific imaging applications for use in detecting or treating precancer or early cancer.

Within the target organ sessions, keynote speakers consider the epidemiology, causes, mechanisms, and risk models of cancer and its clinical presentation; disease incidence and prevalence; lesion pathology; and standard of care. Also reviewed are ongoing and new clinical protocols involving screening and early detection; treatment intervention (with drugs, surgery, and/or IGI); biomarkers of risk that are prognostic, progressive, and predictive; and anatomic, functional, and molecular imaging.

Roundtable discussions follow the target organ sessions, comprised of speakers and imaging device and pharma experts from industry, academia, National Cancer Institute (NCI) and Food and Drug Administration (FDA). The roundtables cover topics related to the feasibility and utility of developing imaging-based biomarkers to evaluate risk and guide intervention in the target organs. These discussions address high-priority clinical settings, further research, and the development effort needed to qualify corresponding imaging-based biomarkers for clinical use in these settings. Specifically, the participants consider the components of a research plan to develop high priority imaging-based biomarkers. 